\title{
Once-Monthly Continuous Erythropoietin Receptor Activator (C.E.R.A.) in Patients with Hemodialysis- Dependent Chronic Kidney Disease: Pooled Data from Phase III Trials
}

Francesco Locatelli - Gabriel Choukroun - Matt Truman •

Alfons Wiggenhauser · Danilo Fliser

Received: January 8, 2016 / Published online: March 10, 2016

(C) The Author(s) 2016. This article is published with open access at Springerlink.com

\section{ABSTRACT \\ Introduction: Erythropoiesis-stimulating agents and iron are commonly used in patients with chronic kidney disease with the aim of correcting}

Enhanced content To view enhanced content for this article go to http://www.medengine.com/Redeem/ C844F0600C480B6E.

Electronic supplementary material The online version of this article (doi:10.1007/s12325-016-0309-6) contains supplementary material, which is available to authorized users.

F. Locatelli $(\varangle)$

Nephrology, Dialysis and Transplantation

Department, Alessandro Manzoni Hospital, Lecco, Italy

e-mail: f.locatelli@ospedale.lecco.it

G. Choukroun

Nephrology, Dialysis and Transplantation Department, CHU Amiens and University of

Picardie Jules Verne, Amiens, France

M. Truman

Roche Australia, Dee Why, Australia

A. Wiggenhauser

Roche Pharma AG, Medical Affairs,

Grenzach-Wyhlen, Germany

D. Fliser

Department of Internal Medicine IV, Saarland

University Medical Centre, Homburg/Saar,

Germany anemia and maintaining stable hemoglobin levels. We analyzed pooled data from 13 studies with similar designs included in the Umbrella Continuous Erythropoietin Receptor Activator (C.E.R.A.) program to investigate the effects of continuous erythropoiesis receptor activator in clinically relevant subgroups of patients with chronic kidney disease and to determine whether the efficacy and safety outcomes demonstrated in the overall chronic kidney disease population are maintained in specific subgroups.

Methods: Data from 13 Phase III trials set up with similar design were retrospectively pooled for this analysis. Patients with chronic kidney disease who had previously been receiving epoetin or darbepoetin were switched to continuous erythropoiesis receptor activator once-monthly after a 4- to 8-week screening period. Patients entered a 16-week continuous erythropoiesis receptor activator dose-titration period followed by an 8-week evaluation period. In total, 2060 patients were included in the analysis. Subgroups were defined based on: hemoglobin target range [lower (10.0-12.0 g/dL)/upper (10.5-13.0 g/dL)], gender (female/male), age $(<65 / \geq 65)$ baseline $\mathrm{N}$-terminal pro-B-type natriuretic peptide 
levels $(<5000 / \geq 5000)$, cardiovascular risk factors (diabetes/cardiac/vascular/none).

Results: Across all subgroups analyzed, switching from shorter-acting erythropoiesis-stimulating agents to continuous erythropoiesis receptor activator once-monthly maintained stable hemoglobin concentrations in a high proportion of patients (78\%), with only moderate hemoglobin fluctuations and a low number of dose changes. The safety profile across subgroups was as expected based on pre-existing risk factors; observed increases in adverse events were attributable to underlying risk factors rather than study drug.

Conclusions: This retrospective analysis of 13 trials showed that continuous erythropoiesis receptor activator once-monthly maintained stable hemoglobin levels across a number of clinically relevant patient subgroups, including those with higher inherent cardiovascular risk. The safety profile was consistent with that previously established in the chronic kidney disease population.

ClinicalTrials.gov identifiers: NCT00413894/

NCT00545571/NCT00517413/NCT00560404/

NCT00882713/NCT00550680/NCT00576303/

NCT00660023/NCT00717821/NCT00642850/

NCT00605293/NCT00661505/NCT00699348.

Funding: F. Hoffmann-La Roche Ltd, Basel, Switzerland.

Keywords: Anemia, chronic kidney disease; Continuous erythropoiesis; Hemodialysis; Receptor activator; Efficacy; Patient subgroups; Pooled analysis

\section{INTRODUCTION}

Anemia is common in patients with chronic kidney disease (CKD); severe anemia can reduce quality of life, and increase the risk of cardiovascular (CV) events and mortality [1, 2]. Anemia management is central in care for patients with CKD, and treatment with iron and erythropoiesis-stimulating agents (ESAs) is the standard of care for patients on dialysis. Therapeutic goals include correcting anemia and maintaining stable hemoglobin ( $\mathrm{Hb})$ levels. Reduced ESA dose and frequency of administration should also be sought [3, 4]. A recent systematic review and meta-analysis of randomized trials in patients with CKD analyzed data from more than 12,000 patients in 40 trials with the aim of comparing the efficacy and safety of ESAs [5]. The conclusion was that epoetins (epoetin alfa, epoetin beta; darbepoetin alfa, methoxy polyethylene glycol-epoetin beta) were similarly effective for preventing blood transfusion and better than placebo and that currently all ESAs are safe and efficacious with minimal differences between the different formulations in the CKD setting.

Despite well-defined therapeutic goals, maintaining $\mathrm{Hb}$ within the desired range is challenging in patients with CKD: many factors, including iron status and comorbidities, influence the response to treatment. Continuous erythropoiesis receptor activator [C.E.R.A. (methoxy polyethylene glycol-epoetin beta)] [6] has a long half-life $(134 \mathrm{~h})$, a relatively low binding affinity for the erythropoietin receptor and low systemic clearance, allowing once-monthly (QM) dosing, which may be more convenient for patients compared with shorter-acting ESAs [6, 7].

A variety of Phase II and III trials of C.E.R.A. have been conducted in CKD. Data from 13 Phase III trials set up with similar design were pooled for this analysis, each multicenter study set up using similar inclusion and exclusion criteria and trial design to allow analyses to be performed both at the single-study level and using pooled data from multiple studies. Data 
from individual studies in dialysis-dependent patients with CKD have demonstrated that C.E.R.A. QM maintains stable on-target $\mathrm{Hb}$ concentrations with fewer dose adjustments than shorter-acting ESAs [7-16]. Pooling data from similar individual studies allows investigating the efficacy and safety of C.E.R.A. in clinically relevant subgroups of patients with $\mathrm{CKD}$, where underlying risk factors can potentially affect patients' response to treatment.

The present pool comprises 13 similarly designed studies investigating the efficacy and safety of C.E.R.A. QM in 2060 dialysis patients. Studies were conducted in real-life settings across numerous countries between 2007 and 2011. The aim of this analysis was to determine whether the efficacy and safety of C.E.R.A. is affected by $\mathrm{CV}$ risk factors, age, gender, or protocol-defined $\mathrm{Hb}$ target ranges.

\section{METHODS}

For this analysis, data from 13 interventional, open-label, multicenter trials included in the Umbrella C.E.R.A. program (ClinicalTrials.gov identifiers: NCT00413894/NCT00545571/ NCT00517413/NCT00560404/NCT00882713/ NCT00550680/NCT00576303/NCT00660023/ NCT00717821/NCT00642850/NCT00605293/ NCT00661505/NCT00699348) conducted in 404 centers across Brazil, Czech Republic, France, Germany, Greece, Hungary, Italy, Latin America, Morocco, Russia, Spain, Switzerland and Turkey were pooled (Supplementary Table 1). Ten single-arm and three 2-arm randomized trials were run under an Umbrella protocol to ensure similar populations and similar treatment regimens. All patients from the 10 single-arm trials and patients from the C.E.R.A. arm of the three randomized trials have been combined for this Umbrella analysis.

\section{Ethics Statement}

Studies were conducted in accordance with the Declaration of Helsinki of 1964, as revised in 2013, and the protocols were approved by the Institutional Review Boards/local Independent Ethics Committees at each center. Written informed consent was obtained from all participants. Data analyzed in this manuscript are from previously published studies.

\section{Subjects, Inclusion and Exclusion Criteria}

Inclusion and exclusion criteria were similar across studies. Patients ( $\geq 18$ years) with chronic renal anemia on hemodialysis meeting the following criteria were eligible: $\mathrm{Hb}$ concentration within the study's target range, adequate iron status [serum ferritin $>100 \mathrm{ng} / \mathrm{mL}$ and transferrin saturation (TSAT) $>20 \%$ or hypochromic red cells $<10 \%$ ], continuous ESA maintenance therapy with unchanged dosing interval and weekly dose during the previous month, regular long-term dialysis with identical mode of dialysis for at least 3 previous months.

Exclusion criteria were relevant acute or chronic bleeding, or erythrocyte transfusion within the preceding 8 weeks, hemoglobinopathy or known hemolysis, active malignant disease, vitamin B12 or folic acid deficiency, pure red cell aplasia, platelet count $>500 \times 10^{9} / \mathrm{L}$ or $<100 \times 10^{9} / \mathrm{L}$, poorly controlled hypertension, myocardial infarction, stroke, severe/unstable coronary artery disease, severe liver disease during the previous 3 months or severe congestive heart failure (New York Heart Association class IV). 
Table 1 Studies, countries and hemoglobin target ranges included in the pooled analysis

\begin{tabular}{llllll}
\hline Study & NCT number & Country & $\begin{array}{l}\text { Number (\%) } \\
\text { of patients }\end{array}$ & $\begin{array}{l}\text { Hemoglobin target } \\
\text { range (g/dL) }\end{array}$ & $\begin{array}{l}\text { Hemoglobin target } \\
\text { range group }\end{array}$ \\
\hline ML21040 & NCT00642850 & Czech Republic & $155(7.5)$ & $10.0-12.0$ & Lower \\
ML21145 & NCT00717821 & France & $225(10.9)$ & $10.0-12.0$ & Lower \\
ML20752 & NCT00660023 & Hungary & $107(5.2)$ & $10.0-12.0$ & Lower \\
ML21438 & NCT00699348 & Italy & $298(14.5)$ & $10.0-12.0$ & Lower \\
ML21060 & NCT00605293 & Spain & $48(2.3)$ & $10.0-12.0$ & Lower \\
ML21096 & NCT00661505 & Turkey & $102(5.0)$ & $10.0-12.0$ & Lower \\
ML21208 & NCT00560404 & Brazil & $76(3.7)$ & $10.5-12.5$ & Upper \\
ML20952 & NCT00550680 & Greece & $152(7.4)$ & $10.5-12.5$ & Upper \\
ML20881 & NCT00517413 & Latin America & $129(6.3)$ & $10.5-12.5$ & Upper \\
ML21797 & NCT00882713 & Morocco & $182(8.8)$ & $10.5-12.5$ & Upper \\
ML20977 & NCT00576303 & Russia & $178(8.6)$ & $10.5-12.5$ & Upper \\
ML20572 & NCT00413894 & Germany & $344(16.7)$ & $11.0-12.5$ & Upper \\
ML20826 & NCT00545571 & Switzerland & $64(3.1)$ & $11.0-13.0$ & Upper \\
\hline
\end{tabular}

Individual study results are summarized as supporting information (Supplementary Table 1) and accessible on http://www. roche-trials.com

\section{Study Design}

Patients with chronic renal anemia on dialysis (Table 1) receiving ESA treatment entered a 4- to 8-week screening period, during which mean $\mathrm{Hb}$ concentrations were maintained within the study's target range (10-12 or $10.5-13 \mathrm{~g} / \mathrm{dL})$. Patients then entered a 16-week C.E.R.A. dose-titration period followed by an 8-week evaluation period (Fig. 1), with $\mathrm{Hb}$ concentrations assessed during screening, titration and evaluation. Subgroups were defined based on: $\mathrm{Hb}$ target range (lower, 10.0-12.0 g/dL/upper, 10.5-13.0 g/dL); gender $(\mathrm{F} / \mathrm{M}) ; \quad$ age $\quad(<65 / \geq 65) ; \quad$ baseline $\mathrm{N}$-terminal pro-B-type natriuretic peptide (NT-proBNP) levels $(<5000 / \geq 5000$, a $5000 \mathrm{ng} / \mathrm{mL}$ cut-off was predictive for various endpoints [17]); CV risk

\begin{tabular}{|c|c|c|}
\hline $\begin{array}{c}\text { ESA } \\
\text { 1-3x/week }\end{array}$ & $\begin{array}{l}\text { C.E.R.A } \\
\text { monthly }\end{array}$ & $\begin{array}{l}\text { C.E.R.A } \\
\text { monthly }\end{array}$ \\
\hline $\begin{array}{l}\text { 4-8-week } \\
\text { Screening }\end{array}$ & $\begin{array}{l}\text { 16-week } \\
\text { Titration }\end{array}$ & $\begin{array}{c}\text { 8-week } \\
\text { Evaluation }\end{array}$ \\
\hline
\end{tabular}

Fig. 1 Common study design. In all 13 studies, enrolled patients entered a 4 - to 8 -week screening period followed by a 16-week C.E.R.A. dose-titration period, and an 8-week evaluation period. C.E.R.A. continuous erythropoietin receptor activator, $E S A$ erythropoiesis-stimulating agents

factors (diabetes/cardiac/vascular/none). Patients were further subdivided per quintiles of C.E.R.A. dose ( $\mu \mathrm{g}: \mathrm{q} 1 \leq 70 ; \mathrm{q} 2=70-110 ; \mathrm{q} 3=110-125$; $\mathrm{q} 4=125-185 ; \mathrm{q} 5 \geq 185), \mathrm{Hb}(\mathrm{g} / \mathrm{dL}: \mathrm{q} 1 \leq 10.65$; q2 $=10.65-11.2 ; \quad$ q3 $=11.2-11.7 ; \quad$ q $4=11.7-$ $12.25 ; \quad \mathrm{q} 5 \geq 12.25)$, and $\mathrm{C}$-reactive protein (CRP) $\quad(\mathrm{mg} / \mathrm{L}: \quad \mathrm{q} 1 \leq 2.26 ; \quad \mathrm{q} 2=2.26-4.14 ;$ $\mathrm{q} 3=4.14-7.21 ; \mathrm{q} 4=7.21-14 ; \mathrm{q} 5 \geq 14)$. 


\section{Study Treatment}

Patients continued to receive epoetin or darbepoetin during screening, with no dose interval changes. C.E.R.A. (Micera ${ }^{\circledR}, \quad$ F. Hoffmann-La Roche Ltd., Basel, Switzerland) was administered during dose titration and evaluation. The starting C.E.R.A. dose was based on the last dose of previous ESA, according to the Summary of Product Characteristics: $<8000$ international units (IU) epoetin or $<40 \mu \mathrm{g}$ darbepoetin alfa $=120 \mu \mathrm{g}$ (or $125 \mu \mathrm{g}$ in study ML20572) C.E.R.A.; 8000-16,000 IU epoetin or $40-80 \mu \mathrm{g}$ darbepoetin alfa $=200 \mu$ g C.E.R.A.; $>16,000$ IU epoetin or $>80 \mu \mathrm{g}$ darbepoetin alfa $=360 \mu$ g C.E.R.A. [18].

C.E.R.A. doses were adjusted during titration and evaluation at the investigator's discretion to maintain $\mathrm{Hb}$ within the pre-defined target range of each individual study.

\section{Efficacy and Safety Comparisons in Defined Subgroups}

Efficacy endpoints were $\mathrm{Hb}$ concentration, $\mathrm{Hb}$ fluctuation, proportion of patients maintaining $\mathrm{Hb}$ stability (Hb concentration change $\leq 1.0 \mathrm{~g} / \mathrm{dL}$ from screening to evaluation period or maintained within the target range), required dose of C.E.R.A. and dose adjustments.

Safety endpoints for comparative subgroup analyses were the number of adverse events (AEs) and serious AEs (SAEs), cardiac events and serious CV events, thromboembolic events (including vascular access thrombosis), hypertensive events (including reports of hypertension, hypertensive crisis and blood pressure fluctuation) and vascular disorders (without hypertensive events) e.g., stenosis, phlebitis, arteriosclerosis.

At each study visit, routine laboratory measurements were conducted. Relevant tests known to correlate with ESA response were: transferrin saturation, ferritin, and CRP. Additionally, an ESA resistance index was computed: the rank of the cumulative ESA dosing in the screening period (before switching to C.E.R.A.) over the rank of the average $\mathrm{Hb}$ during the screening period $[19,20]$. In most studies NT-proBNP was measured at baseline.

\section{Statistical Methods}

This analysis included patients who reached the efficacy evaluation period (intention-to-treat completers).

Average $\mathrm{Hb}$ concentrations for a particular period were based on all $\mathrm{Hb}$ assessments during that period. If $H_{0}, \ldots, H_{n}$ are taken at timepoints $t_{0}, \ldots, t_{n}$, the time-adjusted average $\mathrm{Hb}$ value per patient (Hb concentration) was calculated by:

$$
\text { Hb level }=\frac{1}{2\left(t_{n}-t_{0}\right)} \sum_{i}\left(H_{i}+H_{i-1}\right)\left(t_{i}-t_{i-1}\right)
$$

$\mathrm{Hb}$ fluctuation was estimated by a successive variation measure according to the following formula:

Hb fluctuation

$$
=\sqrt{\frac{1}{2\left(t_{n}-t_{0}\right)} \sum_{i=1}^{n}\left(H_{i}-H_{i-1}\right)^{2} \cdot\left(t_{i}-t_{i-1}\right)}
$$

This fluctuation measure is less prone to overall trends in $\mathrm{Hb}$ development compared with usual standard deviation (SD) measures.

Overall $\mathrm{Hb}$ stability was the proportion of patients maintaining $\mathrm{Hb}$ concentration within $\pm 1.0 \mathrm{~g} / \mathrm{dL}$ from the screening to the evaluation period or staying within target range of the pertaining study.

Differences between subgroups were tested by $t$ tests or Wilcoxon rank sum tests for metric variables or Chi-square tests for categorical variables. 
A word of statistical caution: this study's database is large; therefore even small differences between groups can become statistically significant. Differences should be judged by their relative size and potential clinical relevance, not by formal statistical significance. In order to avoid spurious significance, $p$ values $<0.01$ were considered statistically significant.

\section{RESULTS}

\section{Study Population}

In total, 2060 patients [mean (SD) age 60.6 (15.6) years, $57.6 \%$ male] from 13 studies were included in the analysis. Demographic and baseline characteristics are summarized in Table 2. The largest renal disease etiology subgroups were hypertension $[n=487$ (28\%)], diabetes $\quad[n=351 \quad(21 \%)] \quad$ and glomerulonephritis $\quad[n=318 \quad(19 \%)]$ (percentages based on patients with defined etiology). In total, 1508 patients (73\%) had $\geq 1$ prior disease/risk factor, the most common being vascular disorders (68\%) and metabolism/nutrition disorders (38\%). Most patients $(81 \%)$ received epoetin during screening; $43 \%$ received epoetin alfa, 38\% epoetin beta, and $28 \%$ darbepoetin. Some patients had a change in ESA medication during the screening period, thus numbers add to over $100 \%$.

Patients were divided into subgroups as follows: lower/upper $\mathrm{Hb}$ target range $(n=935$ / $n=1125) ; \quad$ male/female $\quad(n=1186 / n=874)$; $<65 / \geq 65$ years $\quad(n=1090 / n=932) ; \quad$ low $/$ high baseline NT-proBNP $(n=975 / n=624)$; patients with diabetes $(n=535)$, with cardiac risk factors $(n=565)$, with vascular risk factors $(n=1675)$, with no CV risk factors $(n=283)$.
Table 2 Demographic characteristics of patients $(n=2060)$

Male, $n(\%)$

1186

Mean age, years $\pm S D$

$61 \pm 15.6$

Mean weight, $\mathrm{kg} \pm \mathrm{SD}$

$71 \pm 15.2$

Mean height, $\mathrm{cm} \pm \mathrm{SD}$

$166 \pm 9.7$

Ethnicity, $n$ (\%)

White

$1533(74)$

Hispanic or Latino

$53(3)$

Black

Other

Unknown

$445(22)$

Etiology of renal disease, $n(\%)$

Hypertension/large vessel disease

Glomerulonephritis

Diabetes

Interstitial nephritis/pyelonephritis

217 (13)

Polycystic kidney disease

109 (6)

Sec. glomerulonephritis/vasculitis

$56(3)$

Other hereditary/congenital diseases

Neoplasms/tumors

34 (2)

Other

Undefined etiology

Prior diseases/risk factors

Vascular disorders

$1397(68)$

Metabolism and nutrition disorders

Cardiac disorders

390 (19)

Surgical and medical procedures

Nervous system disorders

$151(7)$

Gastrointestinal disorders

$110(5)$

Infections and infestations

29 (1)

Respiratory, thoracic and mediastinal

19 (1)

disorders 
Table 2 continued

ESA administration during screening, \%

$\begin{array}{ll}\text { Epoetin alfa } & 43 \\ \text { Epoetin beta } & 38 \\ \text { Darbepoetin alfa } & 28 \\ \text { Other } & 2\end{array}$

$E S A$ erythropoiesis-stimulating agent, SD Standard deviation

\section{Efficacy}

As seen previously [6, 7], the overall population achieved stable $\mathrm{Hb}$ concentrations throughout screening, titration and evaluation periods, and exhibited $\mathrm{Hb}$ fluctuations $\sim 0.5 \mathrm{~g} / \mathrm{dL}$ during evaluation. The mean $\mathrm{Hb}$ level was $11.4 \mathrm{~g} / \mathrm{dL}$ during both screening and evaluation and was $11.7 \mathrm{~g} / \mathrm{dL}$ during titration. A high proportion of patients exhibited $\mathrm{Hb}$ stability $(\mathrm{Hb}$ concentration within $\pm 1 \mathrm{~g} / \mathrm{dL}$ from screening or within the target range) (Fig. 2). Overall, patients required an average C.E.R.A. dose of $133.4 \mu \mathrm{g}$ during titration and $131.3 \mu \mathrm{g}$ during evaluation. During titration, patients received 8220 C.E.R.A. administrations in total; dose changes were required in 3078 cases (37.4\%). During the evaluation period, patients received a total of 4103 doses; dose changes were required in 737 cases (18.0\%). Efficacy within different subgroups during the evaluation period is considered in detail below.

\section{Hemoglobin Target Range}

Mean achieved $\mathrm{Hb}$ in the lower and upper $\mathrm{Hb}$ target groups differed by $0.4 \mathrm{~g} / \mathrm{dL}$ (11.2 vs 11.6 ; $p<0.0001$ ) (Table 3; Fig. 3). Hb fluctuations were also higher in the upper Hb group (0.46 vs $0.50 ; p=0.001$ ) (Table 3 ), whereas $\mathrm{Hb}$ stability or dose changes did not differ significantly between target range groups.

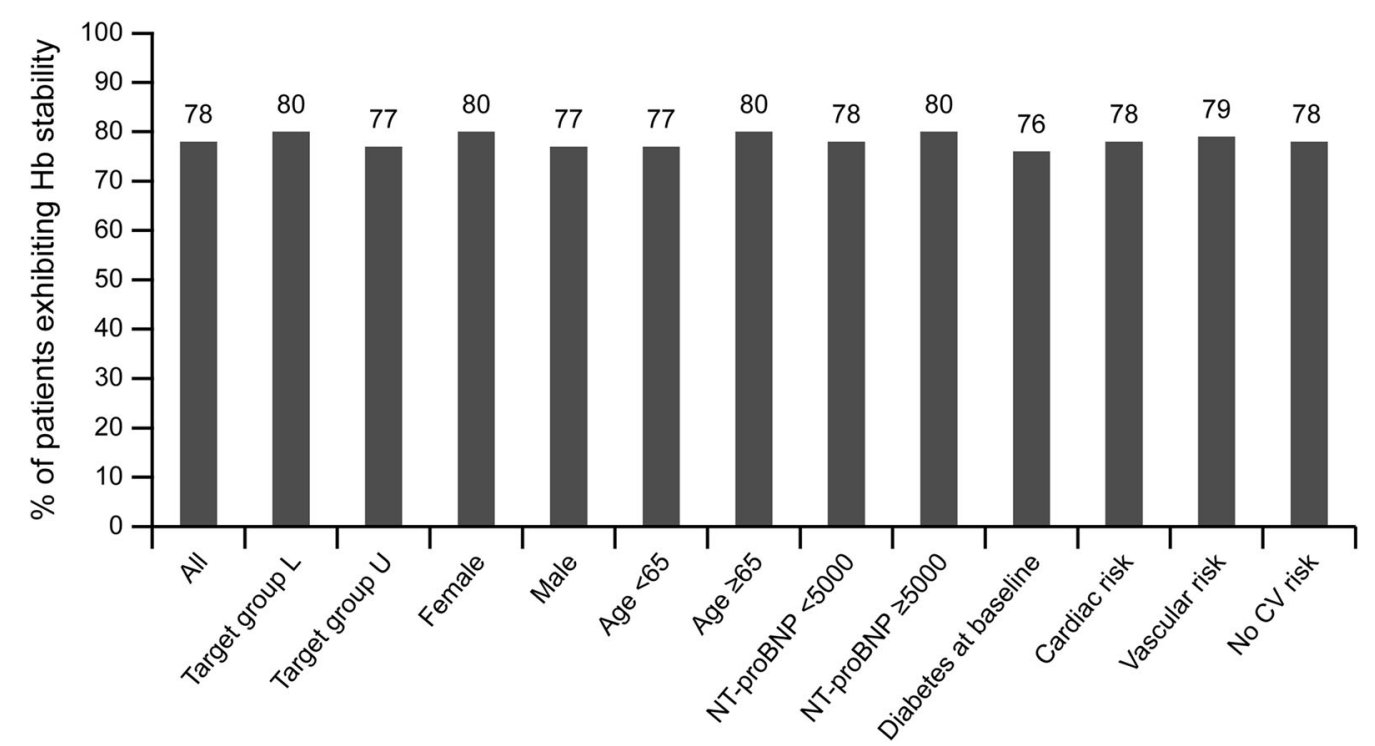

Fig. 2 Proportion of patients exhibiting hemoglobin stability. Across all studied subgroups, a large majority of patients (76-80\%) exhibited hemoglobin stability (hemoglobin concentration within $\pm 1 \mathrm{~g} / \mathrm{dL}$ from screening or within the target range, evaluation period). $C V$ cardiovascular, $L$ lower, NT-proBNP N-terminal pro-B-type natriuretic peptide, $U$ upper 
Table 3 Efficacy comparisons between subgroups during evaluation

\begin{tabular}{|c|c|c|c|c|c|c|c|}
\hline & $\begin{array}{l}\text { Number } \\
\text { of patients }\end{array}$ & $\begin{array}{l}\text { Hemoglobin } \\
(\mathrm{g} / \mathrm{dL})\end{array}$ & $\begin{array}{l}\text { Hemoglobin } \\
\text { fluctuation } \\
(\mathrm{g} / \mathrm{dL})\end{array}$ & $\begin{array}{l}\text { Overall } \\
\text { stability } \\
(\%)\end{array}$ & $\begin{array}{l}\text { Average } \\
\text { monthly dose } \\
(\mu \mathrm{g})\end{array}$ & $\begin{array}{l}\% \text { dose } \\
\text { changes }\end{array}$ & $\begin{array}{l}\text { Dose changes } \\
\text { per patient }\end{array}$ \\
\hline All & 2060 & 11.4 & 0.48 & 78 & 131.3 & 18.0 & 0.4 \\
\hline $\begin{array}{l}\text { Target group } \\
\text { L }\end{array}$ & 935 & 11.2 & 0.46 & 80 & 124.7 & 19.1 & 0.4 \\
\hline $\begin{array}{l}\text { Target group } \\
\text { U }\end{array}$ & 1125 & 11.6 & $0.50^{\mathrm{b}}$ & 77 & $136.9^{\mathrm{b}}$ & 17.1 & 0.3 \\
\hline Female & 874 & 11.4 & 0.48 & 80 & 131.0 & 17.2 & 0.3 \\
\hline Male & 1186 & 11.5 & 0.48 & 77 & 131.6 & 18.5 & 0.4 \\
\hline Age $<65$ & 1090 & 11.5 & 0.5 & 77 & 131.5 & 18.4 & 0.4 \\
\hline Age $\geq 65$ & 932 & 11.4 & $0.45^{\mathrm{a}}$ & 80 & 129.3 & 17.5 & 0.4 \\
\hline $\begin{array}{l}\text { NT-proBNP } \\
\quad<5000\end{array}$ & $975^{\mathrm{e}}$ & 11.4 & 0.47 & 78 & 124.3 & 18.1 & 0.4 \\
\hline $\begin{array}{l}\text { NT-proBNP } \\
\geq 5000\end{array}$ & $624^{\mathrm{e}}$ & 11.4 & 0.49 & 80 & $138.0^{c}$ & 18.2 & 0.4 \\
\hline No CV risk & 283 & 11.5 & 0.51 & 78 & 127.8 & 17.7 & 0.4 \\
\hline Cardiac risk & 565 & 11.4 & $0.46^{\mathrm{d}}$ & 78 & 136.2 & 18.0 & 0.4 \\
\hline Vascular risk & 1675 & 11.4 & 0.48 & 79 & 132.2 & 18.1 & 0.4 \\
\hline $\begin{array}{c}\text { Diabetes at } \\
\text { baseline }\end{array}$ & 535 & 11.3 & 0.47 & 76 & 132.5 & 17.4 & 0.3 \\
\hline
\end{tabular}

Italicized values indicate significant differences $(p<0.01)$ between subgroups: ${ }^{a} p<0.001 ;{ }^{b} p=0.001 ;{ }^{c} p=0.002$; ${ }^{\mathrm{d}} p=0.01$

${ }^{\mathrm{e}} \mathrm{NT}$-proBNP was not measured for all patients

$C V$ cardiovascular, $L$ lower, $N T$-proBNP $\mathrm{N}$-terminal pro-B-type natriuretic peptide, $U$ upper

Patients with lower target $\mathrm{Hb}$ range required significantly lower C.E.R.A. doses than the upper $\mathrm{Hb}$ target subgroup (124.7 vs $136.9 \mu \mathrm{g}$; $p<0.001$ ) (Table 3 ). Iron status and CRP values were not significantly different in the two $\mathrm{Hb}$ target groups; they were slightly more favorable in the upper group (data not shown). ESA resistance index and baseline NT-proBNP were also closer to normal values in the upper target group than in the lower: median TSAT $28.8 \%$ vs $27.5 \%(p=0.05)$; median ferritin 456.5 vs $465.0 \mu \mathrm{g} / \mathrm{L} \quad(p=0.43) ; \quad$ median $\quad$ CRP 4.4 vs
$4.7 \mathrm{mg} / \mathrm{L} \quad(p=0.22)$; median ESA resistance index 0.8 vs $1.4 \quad(p<0.001)$; median NT-proBNP (pg/mL) 2982 vs 4047 ( $p<0.001)$.

\section{Gender}

All efficacy outcomes were similar across male and female subgroups (Table 3 ).

Age

Achieved Hb concentrations, Hb stability, dose changes required, or C.E.R.A. dose required were not significantly different in patients 


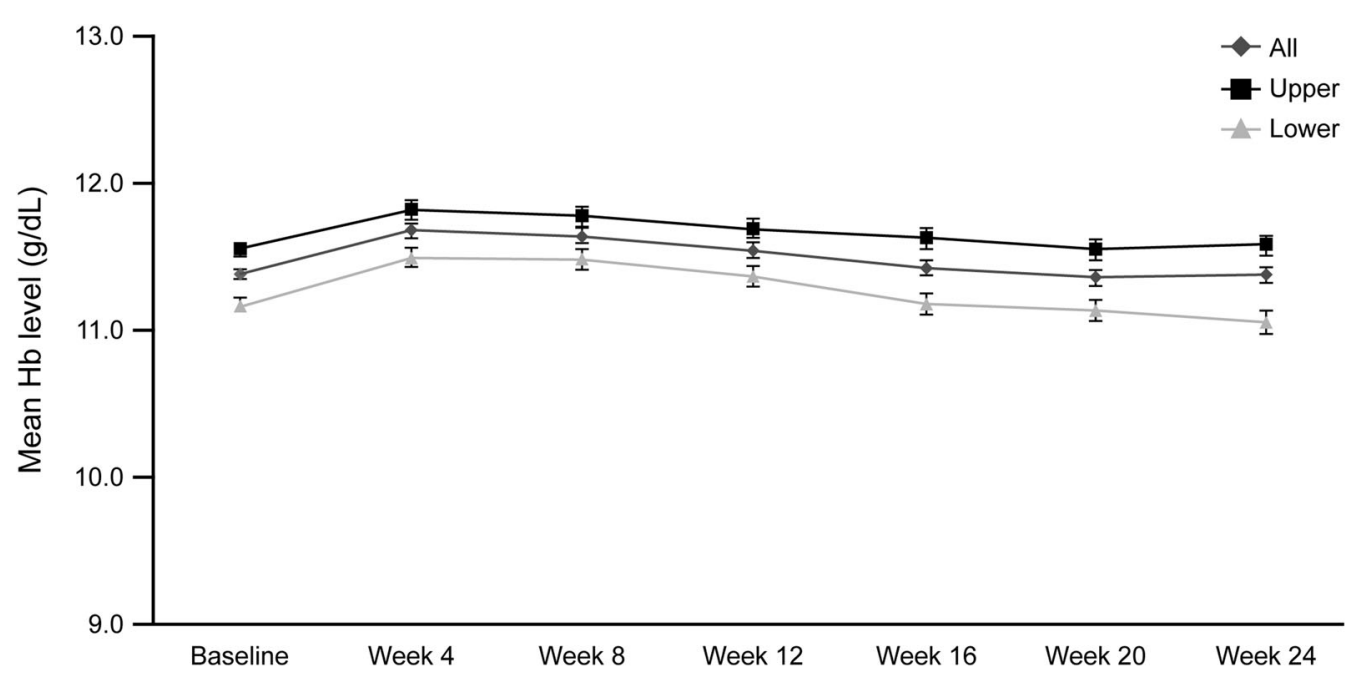

Fig. 3 Mean Hb levels over time, by target Hb group. The error bars show $95 \%$ confidence intervals. In the upper (square symbols) and the lower (triangle symbols) $\mathrm{Hb}$ target groups, the achieved Hb levels were stable over time. Hb hemoglobin

Table 4 Median TSAT, ferritin, CRP and ESA resistance index in subgroups according to NT-proNBP levels high $(>5000 \mathrm{ng} / \mathrm{mL})$ or low $(\leq 5000 \mathrm{ng} / \mathrm{mL})$

\begin{tabular}{lccc}
\hline Variable & \multicolumn{2}{l}{ Baseline NT-proNBP level } & $p$ value \\
\cline { 2 - 3 } & $\begin{array}{l}\text { Low } \\
n=975\end{array}$ & $\begin{array}{l}\text { High } \\
\boldsymbol{n}=\mathbf{6 2 4}\end{array}$ & 0.05 \\
\hline TSAT, \% & 29.8 & 28.2 & 0.11 \\
Ferritin, $\mu \mathrm{g} / \mathrm{L}$ & 430.0 & 470.0 & 0.003 \\
CRP, $\mathrm{mg} / \mathrm{L}$ & 4.0 & 5.0 & $<0.001$ \\
ESA resistance index & 1.1 & 1.3 & \\
\hline
\end{tabular}

Italicized values indicate significant differences $(p<0.01)$ between subgroups

$C V$ cardiovascular, $C R P$ C-reactive protein, $E S A$ erythropoiesis-stimulating agent, $N T$-proBNP N-terminal pro-B-type natriuretic peptide, TSAT transferrin saturation

aged $\geq 65$ years and those $<65$ years (Table 3 ). However, $\mathrm{Hb}$ fluctuation values were significantly higher in patients aged $<65$ years (0.51 vs $0.45 \mathrm{~g} / \mathrm{dL} ; p<0.001$ ) (Table 3 ).

\section{Baseline NT-proBNP Levels}

Patients in the high and low NT-proBNP subgroups did not have significantly different $\mathrm{Hb}$ concentrations, $\mathrm{Hb}$ fluctuation, $\mathrm{Hb}$ stability or required dose changes (Table 3). However, those in the low NT-proBNP group required significantly lower C.E.R.A. doses than those in the high group (124.3 vs $138.0 \mu \mathrm{g} ; p=0.002$ ) (Table 3). The iron status was slightly higher in the low NT-proBNP subgroup than in the high subgroup, while CRP values and ESA resistance index were significantly lower (more favorable) in the low NT-proBNP group than in the high group (Table 4).

\section{Cardiovascular Risk Factors}

Achieved $\mathrm{Hb}$ concentrations, $\mathrm{Hb}$ stability, required C.E.R.A. dose and dose changes were not significantly different in patients with 
Table 5 Median TSAT, ferritin, CRP, ESA resistance index and NT-proNBP levels according to CV risk group

\begin{tabular}{llllllll}
\hline & $\begin{array}{l}\text { No CV risk } \\
\boldsymbol{n}=\mathbf{2 8 3}\end{array}$ & $\begin{array}{l}\text { Diabetes } \\
\boldsymbol{n}=\mathbf{5 3 5}\end{array}$ & $\boldsymbol{p}$ value & $\begin{array}{l}\text { Cardiac risk } \\
\text { factors } \\
\boldsymbol{n}=\mathbf{5 6 5}\end{array}$ & $\boldsymbol{p}$ value & $\begin{array}{l}\text { Vascular risk } \\
\text { factors } \\
\boldsymbol{n}=\mathbf{1 6 7 5}\end{array}$ & $\boldsymbol{p}$ value \\
\hline TSAT, \% & 29.6 & 27.1 & 0.008 & 26.5 & $<0.001$ & 28.1 & 0.02 \\
Ferritin, $\mu \mathrm{g} / \mathrm{L}$ & 428.5 & 494.0 & 0.05 & 487.9 & 0.005 & 465 & 0.07 \\
$\mathrm{CRP}, \mathrm{mg} / \mathrm{L}$ & 4.0 & 5.0 & 0.1 & 5.1 & 0.03 & 4.6 & 0.51 \\
ESA resistance index & 1.2 & 1.0 & 0.02 & 0.9 & $<0.001$ & 1.0 & 0.04 \\
NT-proNBP, $\mathrm{pg} / \mathrm{mL}$ & 2544 & 3463 & $<0.001$ & 5477 & $<0.001$ & 3753 & $<0.001$ \\
\hline
\end{tabular}

Italicized values indicate significant differences $(p \leq 0.01)$ between subgroups (risk group versus no CV risk)

$C V$ cardiovascular, CRP C-reactive protein, ESA erythropoiesis-stimulating agent, NT-proBNP N-terminal pro-B-type natriuretic peptide, TSAT transferrin saturation

pre-existing $\mathrm{CV}$ risk factors and patients with no CV risk factors (Table 3). Hb fluctuation values were significantly different between those with pre-existing cardiac risk factors and those without $\mathrm{CV}$ risk factors (0.46 vs $0.51 \mathrm{~g} / \mathrm{dL} ; p=0.01$ ) (Table 3). Furthermore, a trend towards lower dose requirements in the group with no CV risk factors was observed. TSAT values were slightly favorable for the no $\mathrm{CV}$ risk group compared with patients with diabetes, cardiac or vascular complications, whereas the opposite was seen for ferritin (Table 5). Likewise, CRP values were lower for the no $\mathrm{CV}$ risk group compared with those with diabetes, cardiac or vascular complications, while a higher resistance index was seen in the no CV risk group (Table 5). Baseline NT-proBNP levels were significantly lower in the no CV risk group compared with the other subgroups (Table 5).

\section{Safety}

Overall safety results from individual studies have been reported in the respective publications and study reports. This section focusses on differences in safety outcomes between analyzed patient subgroups.
Compared with patients aged $<65$ years, those aged $\geq 65$ years experienced higher incidences of AEs $(59.4 \%$ vs $72.0 \%, p<0.001)$, SAEs $(20.0 \%$ vs $30.8 \%, p<0.001)$, cardiac AEs (4.7\% vs $8.7 \%, p<0.001)$ and thromboembolic AEs $(1.3 \%$ vs $3.4 \%, p=0.003)$ (Table 6). Patients with a baseline NT-proBNP level $\geq 5000$ experienced higher $\mathrm{AE}$ incidences compared with those in the low NT-proBNP group, including all AEs $(59.1 \%$ vs $65.9 \%, p=0.007)$, SAEs $(19.4 \%$ vs $27.4 \%, p<0.001)$, cardiac AEs (3.3\% vs $9.0 \%, p<0.001)$ and serious CV AEs (2.5\% vs $5.3 \%, p<0.005)$ (Table 6). Overall, patients with pre-existing risk factors also experienced higher $\mathrm{AE}$ incidences compared with patients with no CV risk factors (Table 6).

In the two highest C.E.R.A. dose quintiles, and in the two lowest $\mathrm{Hb}$ quintiles, a slight increase in the rate of cardiac AEs was observed (Fig. 4). In the highest dose group, the percentage of patients experiencing serious CV AEs (6.3\%), thromboembolic AEs (5.1\%), and vascular AEs (2.4\%) was higher than in all other dose quintiles. Contrastingly, similar frequencies of serious $\mathrm{CV}$, thromboembolic and vascular AEs were recorded across the first four $\mathrm{Hb}$ quintiles, and lowest values were observed in the fifth quintile; thus, there was no relationship between 
Table 6 Safety outcomes

\begin{tabular}{|c|c|c|c|c|c|c|c|c|}
\hline & $\begin{array}{l}\text { Number of } \\
\text { patients }\end{array}$ & $\begin{array}{l}\text { Any } \\
\text { AE } \\
(\%)\end{array}$ & $\begin{array}{l}\text { Any } \\
\text { SAE } \\
(\%)\end{array}$ & $\begin{array}{l}\text { Cardiac } \\
\text { AE (\%) }\end{array}$ & $\begin{array}{l}\text { Serious } \\
\text { CV-AE } \\
(\%)\end{array}$ & $\begin{array}{l}\text { Thromboembolic } \\
\text { AE (\%) }\end{array}$ & $\begin{array}{l}\text { Hypertensive } \\
\text { AE (\%) }\end{array}$ & $\begin{array}{l}\text { Vascular } \\
\text { AE (\%) }\end{array}$ \\
\hline All & 2060 & 65 & 24.9 & 6.5 & 3.9 & 2.9 & 7.7 & 1.6 \\
\hline $\begin{array}{l}\text { Target Group } \\
\text { L }\end{array}$ & 935 & 66.5 & 24.9 & 5.9 & 4.2 & 2.9 & 8.1 & 2.3 \\
\hline $\begin{array}{l}\text { Target Group } \\
\text { U }\end{array}$ & 1125 & 63.7 & 22.4 & 6.9 & 3.7 & 2.9 & 7.3 & 1.1 \\
\hline Female & 874 & 66.1 & 25.7 & 5.8 & 3.7 & 2.7 & 7.9 & 1.8 \\
\hline Male & 1186 & 64.2 & 24.2 & 6.9 & 4.1 & 3.0 & 7.5 & 1.4 \\
\hline Age $<65$ & 1090 & 59.4 & 20.0 & 4.7 & 3.5 & 1.3 & 7.7 & 1.6 \\
\hline Age $\geq 65$ & 932 & $72.0^{\mathrm{a}}$ & $30.8^{\mathrm{a}}$ & $8.7^{\mathrm{a}}$ & 4.4 & $3.4^{\mathrm{b}}$ & 7.6 & 1.6 \\
\hline $\begin{array}{l}\text { NT-proBNP } \\
<5000\end{array}$ & $975^{\mathrm{f}}$ & 59.1 & 19.4 & 3.3 & 2.5 & 2.5 & 6.9 & 1.1 \\
\hline $\begin{array}{l}\text { NT-proBNP } \\
\geq 5000\end{array}$ & $624^{\mathrm{f}}$ & $65.9^{\mathrm{d}}$ & $27.4^{a}$ & $9.0^{\mathrm{a}}$ & $5.3^{\mathrm{c}}$ & 3.0 & 9.6 & 2.2 \\
\hline No CV risk & 283 & 45.9 & 17.0 & 2.1 & 1.4 & 2.5 & 2.8 & 0.0 \\
\hline Cardiac risk & 565 & $71.7^{\circ}$ & $34.4^{\mathrm{e}}$ & $11.0^{\mathrm{e}}$ & $6.2^{\mathrm{e}}$ & $4.4^{\mathrm{e}}$ & $8.9^{\mathrm{e}}$ & $2.5^{\mathrm{e}}$ \\
\hline $\begin{array}{l}\text { Vascular } \\
\text { risk }\end{array}$ & 1675 & $64.3^{\mathrm{e}}$ & $26.4^{\mathrm{e}}$ & $7.1^{\mathrm{e}}$ & $4.5^{\mathrm{e}}$ & $2.9^{\mathrm{e}}$ & $8.7^{\circ}$ & 2.0 \\
\hline $\begin{array}{l}\text { Diabetes at } \\
\text { baseline }\end{array}$ & 535 & $71.2^{\mathrm{e}}$ & $33.6^{c}$ & $7.3^{\mathrm{e}}$ & $5.4^{\mathrm{e}}$ & $5.4^{\mathrm{e}}$ & $6.4^{\mathrm{e}}$ & 2.1 \\
\hline
\end{tabular}

Italicized values represent differences $(p<0.01)$ between subgroups: ${ }^{\mathrm{a}} p<0.001 ;{ }^{\mathrm{b}} p<0.003 ;{ }^{\mathrm{c}} p<0.005 ;{ }^{\mathrm{d}} p=0.007$; ${ }^{\mathrm{e}} p<0.01$. For cardiac risk, vascular risk and diabetes at baseline the comparison is with no $\mathrm{CV}$ risk

${ }^{\mathrm{f}} \mathrm{NT}$-proBNP was not measured for all patients

$A E$ adverse event, $C V$ cardiovascular, $L$ lower, $N T$-proBNP $\mathrm{N}$-terminal pro-B-type natriuretic peptide, $S A E$ serious AE, $U$ upper

Hb level and rate of CV AEs. Finally, high CRP levels appear to be associated with increased serious, thromboembolic and vascular AEs: thromboembolic AEs occurred in 5.0\% of patients in the two highest quintiles compared with $3.2 \%$ in the other quintiles, with a similar pattern seen for serious AEs (3.9\% vs $2.2 \%)$ and vascular AEs (2.3\% vs $1.1 \%)$.

\section{DISCUSSION}

This retrospective analysis of 13 trials included in the Phase III C.E.R.A. clinical program evaluated the efficacy and safety of C.E.R.A. in clinically relevant patient subgroups of patients with CKD on hemodialysis, confirming and extending the findings of an earlier poster 

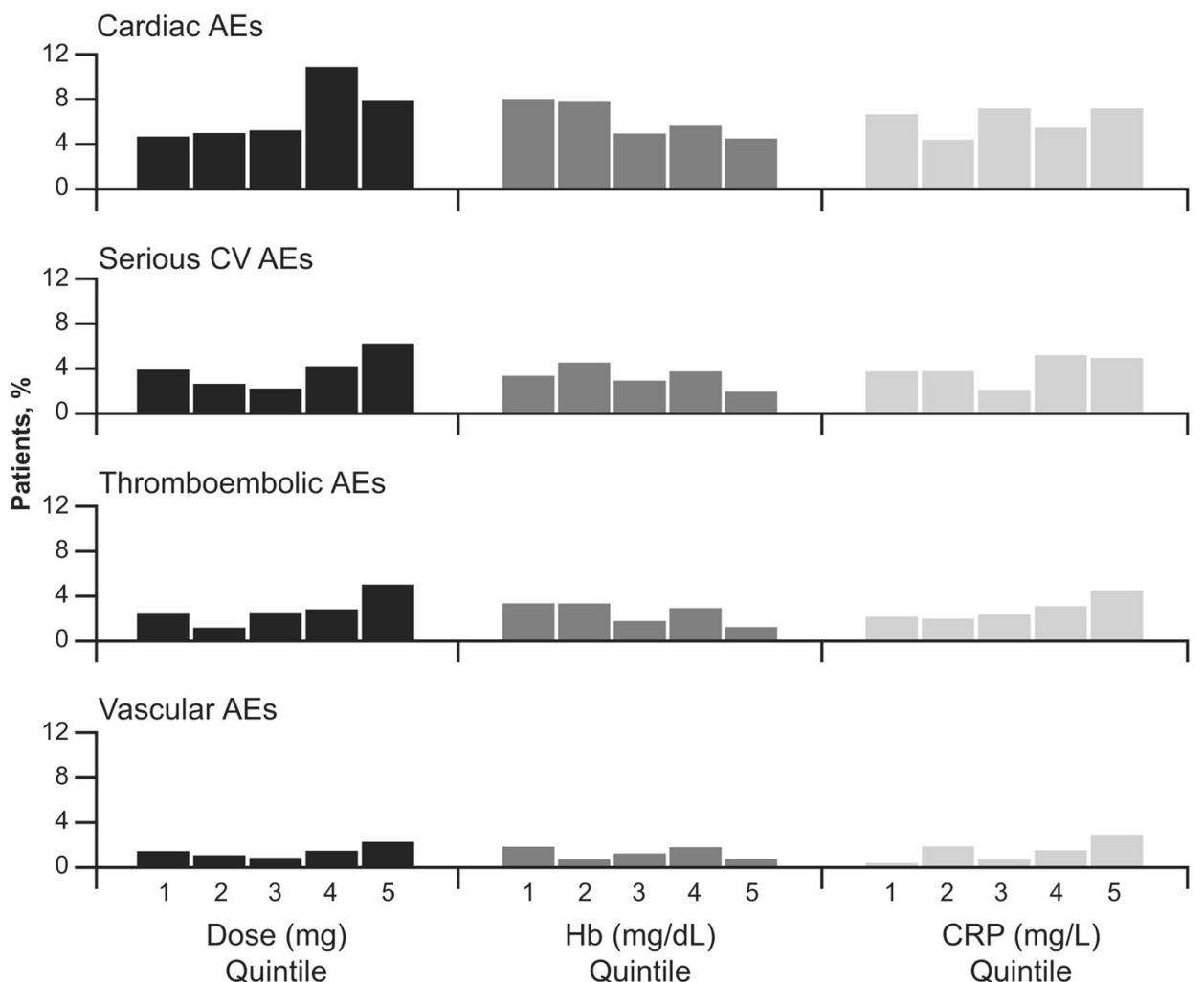

\begin{tabular}{lccccc} 
Quintiles & $\mathbf{1}$ & $\mathbf{2}$ & $\mathbf{3}$ & $\mathbf{4}$ & $\mathbf{5}$ \\
\hline C.E.R.A dose (mg) & $<70$ & $70-110$ & $110-125$ & $125-185$ & $>185$ \\
Hb (g/dL) & $<10.65$ & $10.65-11.2$ & $11.2-11.7$ & $11.7-12.25$ & $>12.25$ \\
CRP (mg/L) & $<2.26$ & $2.26-4.14$ & $4.14-7.21$ & $7.21-14$ & $>14$
\end{tabular}

Fig. 4 Percentage of patients with CV AEs per dose, Hb and CRP quintile. The proportion of patients experiencing cardiac, serious CV, thromboembolic, and vascular AEs is shown by quintiles of $\mathrm{Hb}$, C.E.R.A. dose and CRP. $A E$

presentation of some of these subgroups [22] and an earlier pooled analysis of safety in both dialysis and non-dialysis patients, which concluded that C.E.R.A. showed an overall safety profile comparable with other ESAs [21]. In the current analysis, C.E.R.A. QM maintained stable $\mathrm{Hb}$ concentrations uniformly across all subgroups considered, including those with elevated risk profiles.

The Phase III program included numerous trials of similar design, allowing pooled analysis of results, but the limitations of such an approach should be recognized. All of the analyses were of single arms without a adverse event, C.E.R.A. continuous erythropoietin receptor activator, $C R P$ C-reactive protein, $C V$ cardiovascular, $H b$ hemoglobin

comparator group. While the trials were designed within the same framework as part of an umbrella program, they were performed in different countries and were by no means identical. The post hoc nature of the analysis is also an important limitation, as comparisons were neither pre-planned nor suitably powered to determine significant efficacy and safety differences between subgroups.

Most significant differences between subgroups were numerically small and may not be clinically relevant. The proportion of patients demonstrating $\mathrm{Hb}$ stability was similar across all subgroups, with the lowest value 
(76\%) in patients with diabetes. Mean $\mathrm{Hb}$ fluctuations were around $0.5 \mathrm{~g} / \mathrm{dL}$ across all subgroups, independent of $\mathrm{Hb}$ target, gender and pre-existing $\mathrm{CV}$ risk factors.

The favorable efficacy, safety and tolerability profile of C.E.R.A. in the general CKD population had been reported previously $[7-12,15,16]$. The impact of low $\mathrm{Hb}$ on mortality and CV morbidity was demonstrated in retrospective analyses of nine Phase III, randomized, controlled trials involving 3405 patients with anemic CKD treated with C.E.R.A. [23]. In these analyses, average $\mathrm{Hb}$ level $<10 \mathrm{~g} / \mathrm{dL}$, decrease from stable baseline $\mathrm{Hb}>1 \mathrm{~g} / \mathrm{dL}$, last $\mathrm{Hb}$ level $<10 \mathrm{~g} / \mathrm{dL}$, Hb decline $>1.5 \mathrm{~g} / \mathrm{dL} / 4$ weeks and increased $\mathrm{Hb}$ variability were associated with a higher risk of the composite endpoint and all-cause mortality.

In our analysis, despite stable $\mathrm{Hb}$ maintenance across patient subgroups, there were notable differences in the C.E.R.A. dose required. As expected, patients in the upper $\mathrm{Hb}$ target group required higher doses than those in the lower target group; however, the mean value required for the upper $\mathrm{Hb}$ target group $(136.9 \mu \mathrm{g})$ was relatively low despite the difference being statistically significant. Iron status, CRP, NT-proBNP and ESA resistance index were comparable between subgroups or closer to adequate in the upper target group than the lower, suggesting that dose requirement differences were due to varying target ranges rather than deficiency in ESA-response background variables. The higher doses needed in the high NT-proBNP group compared with the low subgroup may be due to volume overload, hemodilution or underlying diseases such as chronic cardiac congestive failure $[24,25]$. Indeed, high NT-proBNP levels are thought to result from the inflammatory process consequent to cardiac diseases and impaired renal function [25]. Iron status was comparable between the two NT-proBNP groups; however, CRP and ESA resistance index were slightly lower in the low group. Whether these differences can explain the variation in required dose in the two subgroups is uncertain: chronic cardiac congestive failure would not correlate strongly with CRP or iron status. Notably, the proportion of C.E.R.A. dose modifications required was low to moderate across all subgroups.

We acknowledge that caution should be taken when interpreting statistically significant figures that lack clinical plausibility. Likewise, non-significant differences do not provide categorical proof that no difference exists between subgroups. For example, the non-significant trend towards lower dose requirements in no-CV risk patients is supported by elevated NT-proBNP levels in patients with pre-existing $\mathrm{CV}$ risk factors, reinforcing the observation that NT-proBNP levels may be predictive for higher C.E.R.A. dose requirements.

Safety outcomes were as expected across subgroups. Neither $\mathrm{Hb}$ target range nor gender influenced the incidence of AEs; however, all AEs, SAEs, cardiac AEs and serious CV AEs were significantly higher in patients with underlying risk factors (older age, high NT-proBNP or pre-existing $\mathrm{CV}$ risk factors) compared with their lower risk counterparts. Non-significant differences in the incidences of thromboembolic and vascular AEs were also observed between subgroups. Observations in the NT-proBNP subgroups are supported by a recent analysis in end-stage renal disease patients on dialysis, where high baseline NT-proBNP levels predicted higher incidences of cardiac and CV endpoints (5000 ng/mL) [17]. Moreover, elevated NT-proBNP levels have been revealed as an independent mortality predictor in incident hemodialysis patients [25]. While 
baseline NT-proBNP level was not predictive for hypertensive AEs, pre-existing $\mathrm{CV}$ risk factors were. Overall, the increase in CV AEs experienced with pre-existing $\mathrm{CV}$ risk factors suggests AEs were not related to C.E.R.A. administration.

CV AEs of interest were analyzed by quintiles of $\mathrm{Hb}$, C.E.R.A. dose and CRP. A slight increase in $\mathrm{AE}$ rates was observed in the quintiles with lowest $\mathrm{Hb}$ levels and highest doses. These findings agree with previous observations and clinical guidelines that recommend conservative $\mathrm{Hb}$ targets (10-12 g/dL), individualized for each patient's comorbidities. If ESA therapy is used in patients hyporesponsive to ESA treatment, aiming towards the lower $\mathrm{Hb}$ levels of the target range is recommended $[3,26]$.

Furthermore, our data showed that chronic elevation of CRP levels was associated with increased thromboembolic and vascular AEs. This agrees with previous observations that suggest CRP-a marker of inflammationconstitutes an independent risk factor for $\mathrm{CV}$ disease [27]. Caution should be used in patients hyporesponsive to ESA treatment having high CRP levels or in those with specific risk factors (especially diabetics) or conditions such as symptomatic limb arteriopathy, stroke or non-symptomatic ischemic heart disease, or cancer [27].

\section{CONCLUSION}

C.E.R.A. QM maintained stable $\mathrm{Hb}$ concentrations with moderate fluctuations across subgroups of patients with chronic renal anemia on dialysis switching from maintenance therapy with shorter-acting ESAs, including those with underlying risk factors. Differences in required C.E.R.A. doses were observed between patients with upper and lower $\mathrm{Hb}$ target levels, and with high and low baseline NT-proBNP levels. The safety profile across subgroups was as expected based on pre-existing risk factors; any increases in AEs were related to underlying risk factors rather than to the study drug.

\section{ACKNOWLEDGMENTS}

Sponsorship for the studies in this analysis and article processing charges were funded by $\mathrm{F}$. Hoffmann-La Roche Ltd, Basel, Switzerland. All named authors meet the International Committee of Medical Journal Editors (ICMJE) criteria for authorship for this manuscript, take responsibility for the integrity of the work as a whole, and have given final approval to the version to be published. The authors would like to thank Milena Studer, International Scientific Director Mature Products, F. Hoffmann-La Roche Ltd, for critical review of the manuscript. We would also like to acknowledge the editorial assistance provided by the Fishawack Group of Companies and Health Interactions which was supported by F. Hoffmann-La Roche Ltd, Basel, Switzerland.

Disclosures. FL is or was a member of an advisory board and/or speaker at meetings supported with unrestricted grants by AbbVie, Akebia, Amgen, Astellas, Fibrogen, Genzyme, GSK, Janssen, Keryx, MCI, Pharmacosmos, Roche, Takeda, Vifor-Fresenius Pharma, and Z-pharma. GC has received fees as a speaker in meetings sponsored by Roche, Amgen, Novartis, Genzyme; his institution received research grants from Roche and Novartis in the past 5 years. MT and AW are Roche employees. DF has received grant/research support from Janssen-Cilag and Roche Pharma; 
consultant/advisor fees from Amgen, Janssen-Cilag, Roche Pharma, and Vifor; speakers' bureau fees from Amgen, Roche Pharma, and Vifor.

\section{Compliance and Ethics Guidelines. All} procedures followed were in accordance with the ethical standards of the responsible committee on human experimentation (institutional and national) and with the Helsinki Declaration of 1964, as revised in 2013. Informed consent was obtained from all patients for being included in the study. Data analyzed in this manuscript are from previously published studies.

Open Access. This article is distributed under the terms of the Creative Commons Attribution-NonCommercial 4.0 International License (http://creativecommons.org/licenses/ by-nc/4.0/), which permits any noncommercial use, distribution, and reproduction in any medium, provided you give appropriate credit to the original author(s) and the source, provide a link to the Creative Commons license, and indicate if changes were made.

\section{REFERENCES}

1. Portoles J, Gorriz JL, Rubio E, de Alvaro F, Garcia F, Alvarez-Chivas V, et al. The development of anemia is associated to poor prognosis in NKF/KDOQI stage 3 chronic kidney disease. BMC Nephrol. 2013;14:2.

2. Kovesdy CP, Trivedi BK, Kalantar-Zadeh K, Anderson JE. Association of anemia with outcomes in men with moderate and severe chronic kidney disease. Kidney Int. 2006;69:560-4.

3. Locatelli F, Covic A, Eckardt KU, Wiecek A, Vanholder R. Anaemia management in patients with chronic kidney disease: a position statement by the Anaemia Working Group of European Renal Best Practice (ERBP). Nephrol Dial Transpl. 2009;24:348-54.
4. Locatelli F, Del Vecchio L. An expert opinion on the current treatment of anemia in patients with kidney disease. Expert Opin Pharmacother. 2012;13:495-503.

5. Palmer SC, Saglimbene V, Mavridis D, Salanti G, Craig JC, Tonelli $\mathrm{M}$, et al. Erythropoiesis-stimulating agents for anaemia in adults with chronic kidney disease: a network meta-analysis. Cochrane Database Syst Rev. 2014;12:CD010590.

6. Macdougall IC, Robson R, Opatrna S, Liogier X, Pannier A, Jordan P, et al. Pharmacokinetics and pharmacodynamics of intravenous and subcutaneous continuous erythropoietin receptor activator (C.E.R.A.) in patients with chronic kidney disease. Clin J Am Soc Nephrol. 2006;1:1211-5.

7. Locatelli F, Villa G, de Francisco AL, Albertazzi A, Adrogue HJ, Dougherty FC, et al. Effect of a continuous erythropoietin receptor activator (C.E.R.A.) on stable haemoglobin in patients with CKD on dialysis: once monthly administration. Curr Med Res Opin. 2007;23:969-79.

8. Levin NW, Fishbane S, Canedo FV, Zeig S, Nassar GM, Moran JE, et al. Intravenous methoxy polyethylene glycol-epoetin beta for haemoglobin control in patients with chronic kidney disease who are on dialysis: a randomised non-inferiority trial (MAXIMA). Lancet. 2007;370:1415-21.

9. Sulowicz W, Locatelli F, Ryckelynck JP, Balla J, Csiky B, Harris $\mathrm{K}$, et al. Once-monthly subcutaneous $\quad$ C.E.R.A. maintains stable hemoglobin control in patients with chronic kidney disease on dialysis and converted directly from epoetin one to three times weekly. Clin J Am Soc Nephrol. 2007;2:637-46.

10. Dellanna F, Winkler RE, Bozkurt F, Schettler V, Graf $\mathrm{S}$, Bockreiss $\mathrm{N}$, et al. Dosing strategies for conversion of haemodialysis patients from short-acting erythropoiesis stimulating agents to once-monthly C.E.R.A.: experience from the MIRACEL study. Int J Clin Pract. 2011;65:64-72.

11. Weinreich T, Leistikow F, Hartmann HG, Vollgraf G, Dellanna F. Monthly continuous erythropoietin receptor activator treatment maintains stable hemoglobin levels in routine clinical management of hemodialysis patients. Hemodial Int. 2012;16:11-9.

12. Fliser D, Kleophas W, Dellanna F, Winkler RE, Backs W, Kraatz U, et al. Evaluation of maintenance of stable haemoglobin levels in haemodialysis patients converting from epoetin or darbepoetin to monthly intravenous C.E.R.A.: the MIRACEL study. Curr Med Res Opin. 2010;26:1083-9. 
13. Mann JF, de Francisco A, Nassar G, Canaud B. Fewer dose changes with once-monthly C.E.R.A. in patients with chronic kidney disease. Clin Nephrol. 2011;76:9-15.

14. Carrera F, Lok CE, de Francisco A, Locatelli F, Mann JF, Canaud B, et al. Maintenance treatment of renal anaemia in haemodialysis patients with methoxy polyethylene glycol-epoetin beta versus darbepoetin alfa administered monthly: a randomized comparative trial. Nephrol Dial Transpl. 2010;25:4009-17.

15. Bastos K, Lucarelli LA, De Francesco-Daher E, Filho RP, Henriquez C, Espinoza B, et al. C.E.R.A. maintains stable hemoglobin in Latin American patients on dialysis. Int Urol Nephrol. 2012;45:1355-64.

16. Chen N, Qian JQ, Mei CL, Zhang AH, Xing CY, Wang $\mathrm{L}$, et al. The efficacy and safety of continuous erythropoietin receptor activator in dialytic patients with chronic renal anemia: an open, randomized, controlled, multi-center trial. Zhonghua Nei Ke Za Zhi. 2012;51:502-7.

17. Dellanna F, Dickenmann M, Shilo V, Kleophas W. Prognostic value of N-terminal Pro-B-type natriuretic peptide (NT-proBNP) concentrations in patients with end stage renal disease. Proceedings of Kidney Week; 2012 Oct 30-Nov 4; San Diego, CA, USA. American Society of Nephrology; 2012.

18. European Medicines Agency: Mircera Summary of Product Characteristics. 2009 [updated 07/10/2014; cited 04/03/15]. http://www.ema.europa.eu/docs/ en_GB/document_library/EPAR_-_Product_Infor mation/human/000739/WC500033672.pdf. Accessed 4 Mar 2015.

19. Locatelli F, Dusilova-Sulkova S. Characteristics of patients with chronic kidney disease on dialysis with a poor response to continuous erythropoietin receptor activator (C.E.R.A.) once monthly: an analysis of 10 international trials. Nephrol Dial Transpl. 2012;27(suppl 2):ii133-ii145.

20. Fliser D, Shilo V. Continuous erythropoietin receptor activator (C.E.R.A.) once monthly maintains stable hemoglobin values in high-risk patients with chronic kidney disease on dialysis: an analysis of 10 international trials. Nephrol Dial Transpl. 2012;27(suppl 2):ii133-ii145.

21. Locatelli F, Mann JF, Aldigier JC, Sanz Guajardo D, Schmidt R, Van Vlem B, et al. C.E.R.A. safety profile: a pooled analysis in patients with chronic kidney disease. Clin Nephrol. 2010;73:94-103.

22. Locatelli F, Choukroun G, Fliser D, Moecks J, Wiggenhauser A. Dosing of continuous erythropoietin receptor activator (C.E.R.A.) once monthly vs darbepoetin, epoetin alfa or epoetin beta in high-risk patients with chronic kidney disease on dialysis: an analysis of 13 international trials Nephrol Dial Transpl. 2014:29(Suppl 3):iii491-iii500.

23. Locatelli F, de Francisco A, Deray G, Fliser D, Armstrong G, Dougherty FC, et al. Mortality and cardiovascular morbidity associated with haemoglobin levels: a pooled analysis of randomised controlled trials. Nephron Clin Pract. 2014;128:323-32.

24. Locatelli F, Altieri P, Andrulli S, Sau G, Bolasco P, Pedrini LA, et al. Predictors of haemoglobin levels and resistance to erythropoiesis-stimulating agents in patients treated with low-flux haemodialysis, haemofiltration and haemodiafiltration: results of a multicentre randomized and controlled trial. Nephrol Dial Transpl. 2012;27:3594-600.

25. Locatelli F, Hannedouche T, Martin-Malo A, Jacobson SH, Vanholder R, Ronco C, et al. The relationship of NT-proBNP and dialysis parameters with outcome of incident haemodialysis patients: results from the membrane permeability outcome study. Blood Purif. 2013;35:216-23.

26. Locatelli F, Barany P, Covic A, De Francisco A, Del Vecchio L, Goldsmith D, et al. Kidney disease: improving global outcomes guidelines on anaemia management in chronic kidney disease: a european renal best practice position statement. Nephrol Dial Transpl. 2013;28:1346-59.

27. Lagrand WK, Visser CA, Hermens WT, Niessen HW, Verheugt FW, Wolbink GJ, et al. C-reactive protein as a cardiovascular risk factor: more than an epiphenomenon? Circulation. 1999;100:96-102. 